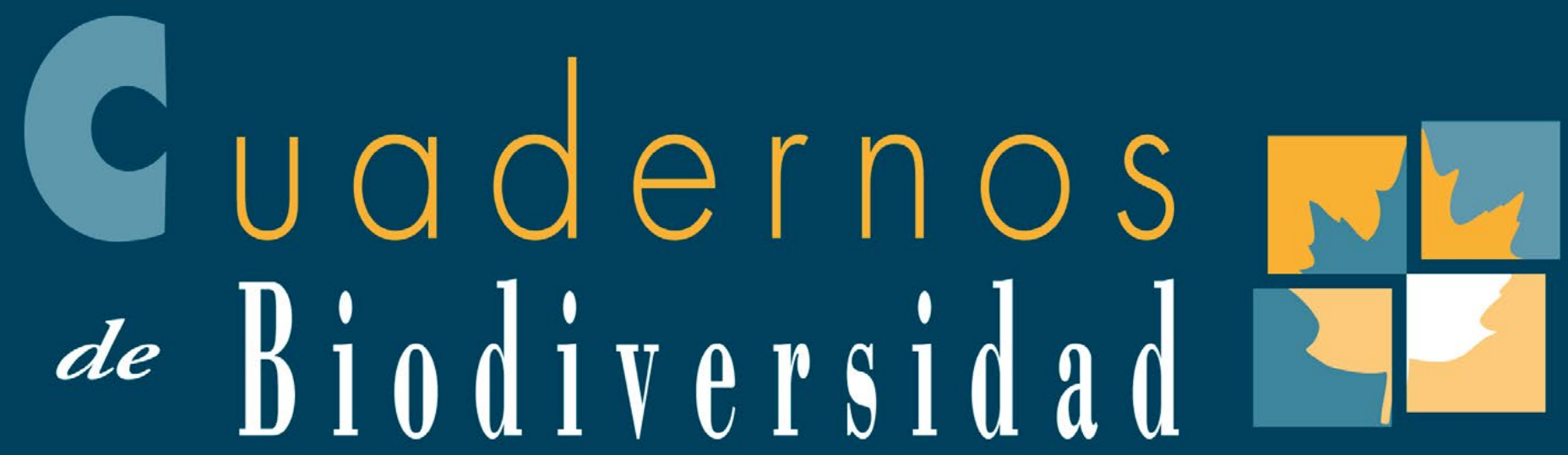

Cuadernos de biodiversidad número 48 • 2015
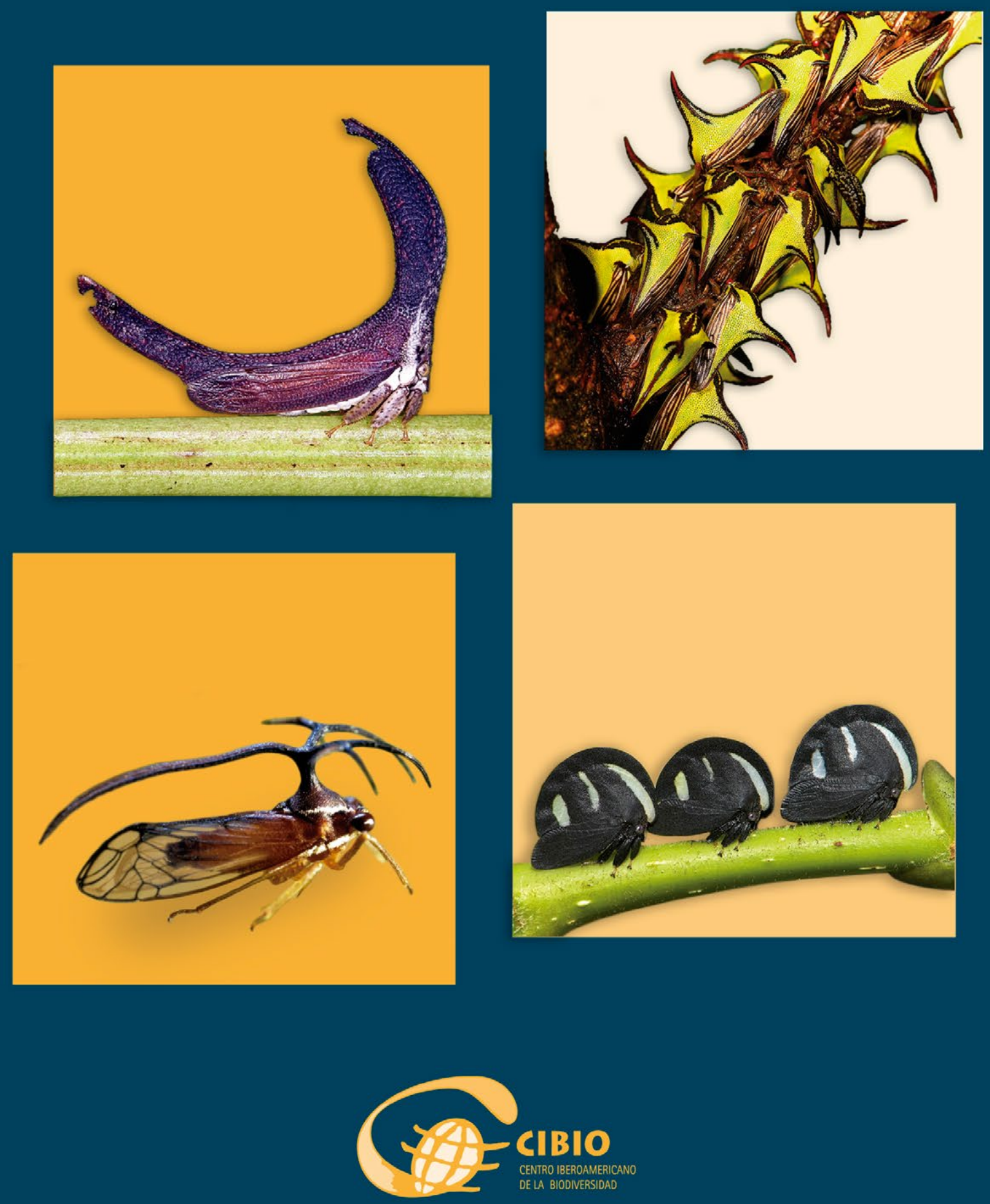

Universitat d'Alacant
Universidad de Alicante 


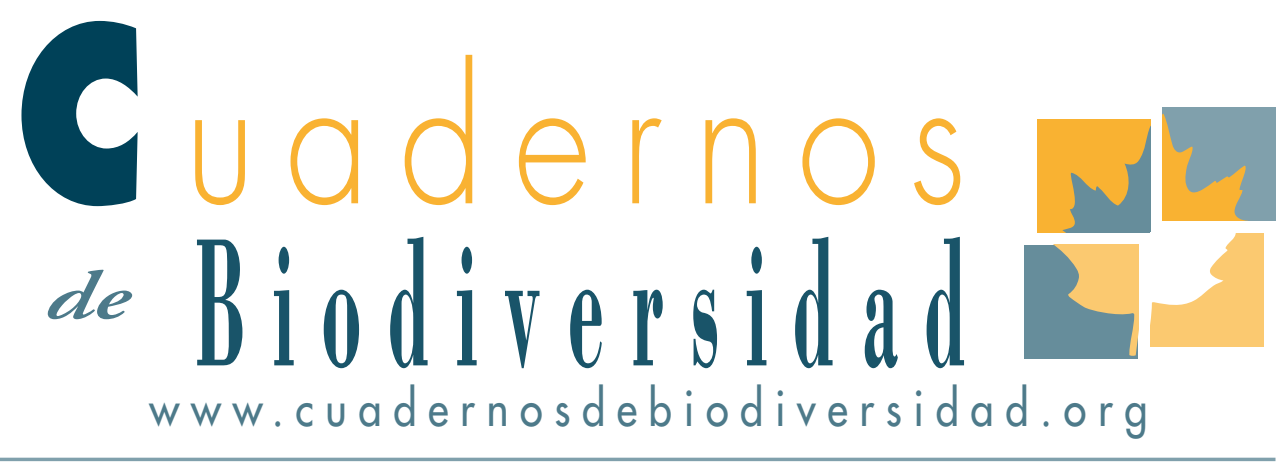

\section{Jugar a extinguir ensambles: importancia de las oquedades de los árboles para la biodiversidad de insectos saproxílicos}

\section{Javier Quinto, Ma Ángeles Marcos-García, Eduardo Galante, Estefanía Micó}

Centro Iberoamericano de la Biodiversidad (CIBIO), Universidad de Alicante, Alicante, EsPAÑA

\section{ABSTRACT}

The assessment of the relation between species diversity, species interactions and stability is a major goal for conservation strategies of saproxylic insects in Iberian Mediterranean woodlands. In these woodlands, tree hollows in standing living trees are key microhabitats for saproxylic biodiversity. We assess the variation in the tree hollow-saproxylic insect network among three woodland sites in the Cabañeros National Park (Spain), and evaluate their implications for saproxylic assemblages' persistence, through simulations of three possible scenarios of loss of tree hollow microhabitats. The composition of tree hollows is closely related to biotic and ecological complexity of saproxylic networks, and together exhibit positive effects on network stability. By other side, different scenarios entail different reorganizations of saproxylic diversity of species and interactions, and the variation of the main interacting attributes. Woodlands providing higher proportion of large tree hollows are less sensitive to microhabitat loss. Conservation strategies for saproxylic insects in Iberian Mediterranean woodlands should consider woodland composition and configuration as key factors in the selection of priority conservation areas.

Key words: Saproxylic insect, tree hollow, Mediterranean woodland, Iberian Peninsula, extinction simulation, network stability. 


\section{INTRODUCCIÓN}

Cuando nos adentramos en un bosque en Europa solemos pensar que estamos ante naturaleza en estado puro. Lejos de ser cierto, los bosques tal y como los conocemos en la actualidad son el resultado de una larga historia de aprovechamiento de los recursos naturales por parte de la especie humana, que ha jugado y continua jugando un extraordinario papel transformador. Como consecuencia de la fragmentación, alteración y reducción de los bosques, la biodiversidad ha experimentado una profunda reestructuración, llevándose la peor parte aquellas especies estrechamente ligadas a los recursos forestales, tales como son los organismos saproxílicos: cualquier especie que depende de la madera o de sus microhábitats asociados en algún estadio de su ciclo biológico (Speight, 1989; Alexander, 2008).

Pese a este escenario de seria amenaza, no es hasta la celebración del Consejo de Europa de 1989 cuando se aborda por primera vez la necesidad de conocer y conservar las comunidades saproxílicas, reconociendo el indiscutible papel ecológico que desempeñan en los ecosistemas como degradadores de la materia orgánica (mayoritariamente los estadios inmaduros) y como polinizadores de las plantas de los bosques (los estadios adultos de muchas especies, como los Syrphidae), y señalándolos también como importantes bioindicadores del estado de conservación de los bosques (Speight, 1989). A partir de entonces se genera un clima favorable para la creación de un marco legal europeo de protección de la fauna saproxílica y de sus hábitats, y se fomenta la inclusión de especies saproxílicas en listados nacionales e internacionales de especies amenazadas (ej. Nieto \& Alexander, 2010). A pesar de esto, la cruda realidad es que las comunidades saproxílicas continúan hoy estando amenazadas, pues aún padecen una alarmante reducción de sus poblaciones y de sus hábitats.

Las principales amenazas de los bosques mediterráneos de la Península Ibérica, y por lo tanto de la biodiversidad saproxílica que éstos encierran, son los incendios forestales, el sobrepastoreo y los cambios de uso de suelo como el abandono de las actividades tradicionales (Haslett, 2007). Por otra parte, la necesidad de conservar las comunidades saproxílicas también se ha hecho patente en España, a través de la incorporación de varias de sus especies en el Libro Rojo de Invertebrados de España (Verdú \& Galante, 2006; Marcos-García \& Quinto, 2011). Asimismo, el incremento del número de proyectos de investigación dedicados al estudio de estas comunidades es reflejo de esta creciente preocupación y sensibilización. Sin embargo, el estudio de la fauna saproxílica en los bosques mediterráneos ibéricos, de los procesos biológicos y ecológicos asociados, y de las relaciones entre sus especies sigue siendo todo un reto al conocimiento de nuestro patrimonio natural, ya que únicamente se han realizado algunos estudios en áreas naturales concretas y de grupos taxonómicos particulares, principalmente dedicados a las especies que figuran en la Directiva Hábitat.

\section{LOS INSECTOS SAPROXÍLICOS DEL PAR- QUE NACIONAL DE CABAÑEROS}

El Parque Nacional de Cabañeros es una de las áreas naturales más representativas, mejor conservadas y más estudiadas del monte mediterráneo en España. Desde la perspectiva de la biodiversidad de insectos saproxílicos, puede considerarse el área natural mejor conocida de la Península Ibérica (Micó et al., 2013).

Cabañeros comprende un mosaico heterogéneo de tipos de vegetación, donde las formaciones boscosas aparecen salpicadas dentro de una matriz herbáceo-arbustiva predominante. Cada uno de sus bosques mediterráneos atesora un singular elenco de insectos saproxílicos (Micó et al., 2013; García-López et al., 2014), cuya riqueza y exclusividad de especies depende en gran medida de la heterogeneidad y abundancia de microhábitats y de recursos tróficos, factores asociados con el grado de madurez del bosque (Quinto et al., 2014). Sin lugar a dudas, las oquedades arbóreas albergan una de las comunidades más ricas e interesantes de los bosques de Cabañeros (Marcos-García et al., 2010; Quinto et al., 2013), dando además cobijo a seis especies de insectos saproxílicos con distinto grado 
de amenaza según criterios de la UICN (Nieto \& Alexander, 2010).
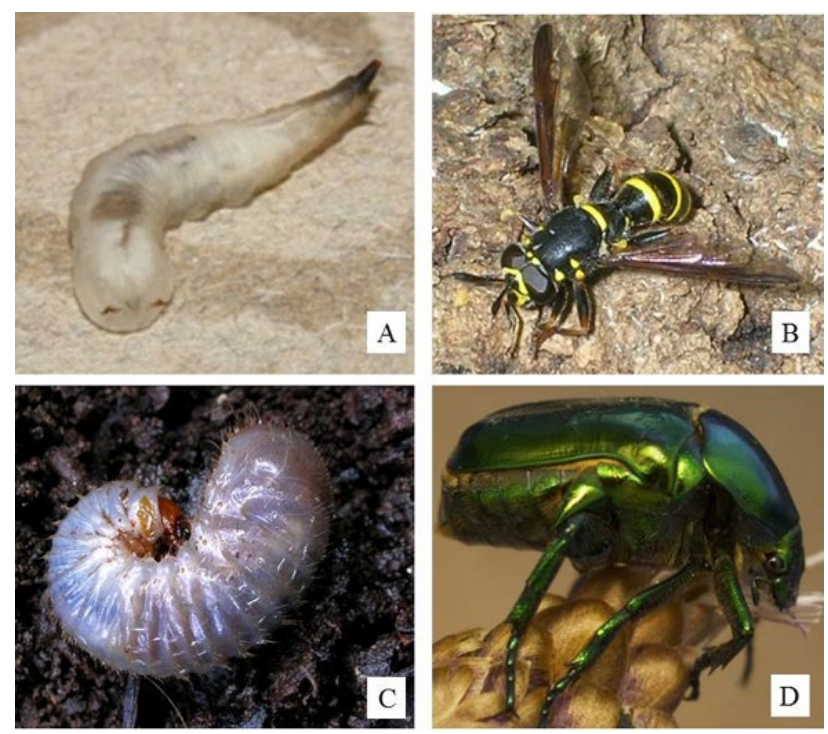

Figura 1. Larvas y adultos de Diptera Syrphidae y Coleoptera. A: Larva de sírfido del género Callicera (Foto: MA.Marcos-García); B: Adulto de Sphiximorpha subsessilis (Foto: Z.Nedeljković); C: Larva de Cetoniidae (Foto: E.Micó); D: Adulto de Cetonia aurataeformis (Foto: J.Ordóńez).

Durante un año, realizamos un estudio de la fauna saproxílica de Coleoptera y Diptera: Syrphidae (Figura 1) en tres de los bosques mediterráneos más representativos del Parque Nacional de Cabañeros (España). Seleccionamos un robledal mixto de melojo (Quercus pyrenaica) y quejigo ( $Q$. faginea), una fresneda (Fraxinus angustifolia), y un encinar (Q. rotundifolia) (Figura 2), considerando 30, 27 y 30 oquedades, respectivamente. Como método de muestreo empleamos trampas de emergencia, que permiten recolectar, a medida que van naciendo, los insectos saproxílicos que se desarrollan en el interior de las oquedades (Figura 3). La diversidad de coleópteros saproxílicos está compuesta por 155 especies pertenecientes a 39 familias, y la de los dípteros sírfidos por 22 especies.

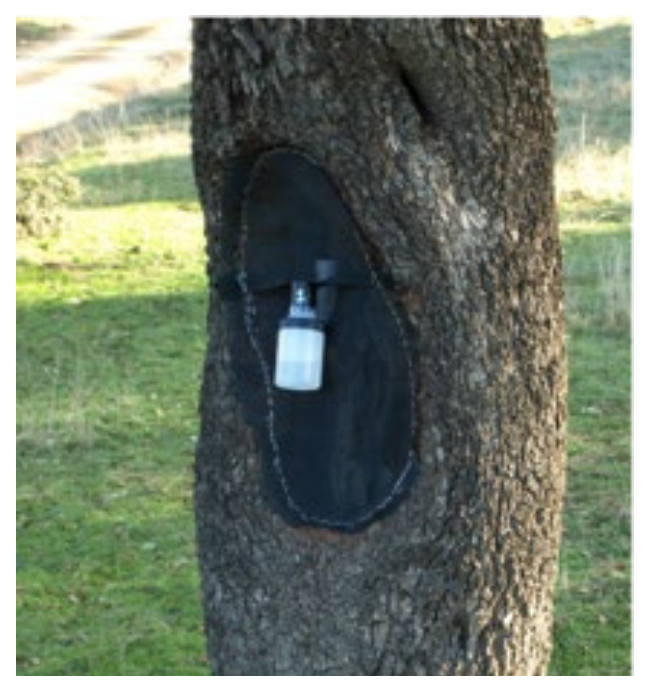

Figura 3. Modelo de trampa de emergencia (Foto: J.Ordóńez).

\section{SIMULAR EXTINCIONES}

En la última década ha tenido lugar un gran avance en las técnicas de análisis de redes ecológicas complejas: aquellas compuestas por un alto número de especies interactuantes. Entre los aspectos más interesantes de esta metodología se encuentra la posibilidad de explorar los patrones de interacción planta-animal, así como la simulación de distintos escenarios de extinción de especies. Esto ha abierto
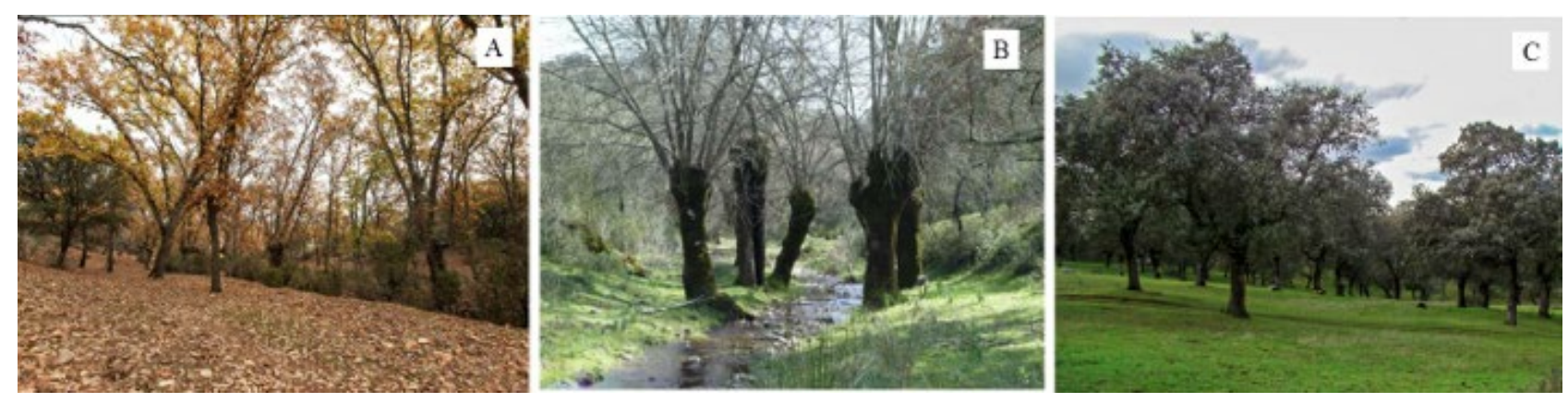

Figura 2. Bosques mediterráneos estudiados en Cabañeros. A: Robledal mixto, B: Fresneda, C: Encinar (Foto: E.Micó, MA.Marcos-García, E.Micó, respectivamente). 
la puerta al estudio de los patrones de interacción o concurrencia entre oquedades e insectos saproxílicos en los bosques mediterráneos de Cabañeros, así como la evaluación de la capacidad de sobrevivir de los saproxílicos ante la desaparición simulada de las oquedades de las que dependen estrechamente (Quinto et al., 2012; Quinto et al., 2015).

Cuando estudiamos redes ecológicas, nos fijamos en atributos de interacción como: 1) número y abundancia de especies que componen cada nivel trófico, 2) número medio de enlaces (cada una de las incidencias de cada especie saproxílica en las oquedades dividido por el número total de especies), 3) la conectividad (proporción de enlaces realizados sobre el total de enlaces posible) y 4) la densidad de enlaces (promedio de abundancia de interacciones), que pueden ser obtenidos empleando un paquete computacional llamado 'bipartite' en el lenguaje de programación $\mathrm{R}$ (Proyecto $\mathrm{R}$ para análisis estadístico) (Dormann et al., 2008). También a través de 'bipartite' se pueden simular los distintos escenarios de eliminación de microhábitats, ya sea eliminando las oquedades más conectadas (aquellas que albergan un mayor número de especies saproxílicas diferentes), las menos conectadas (aquellas oquedades más pobres en especies saproxílicas), o bien realizar una eliminación aleatoria de oquedades.

\section{ENFOQUE DE LOS RESULTADOS}

Los bosques con árboles más viejos, en este caso el robledal mixto y la fresneda, presentan un mayor número de oquedades voluminosas, lo que repercute en la complejidad de los patrones de interacción oquedad-saproxílico. Las oquedades voluminosas son sistemas multi-hábitat, ya que simultáneamente contienen una gran variedad y abundancia de micrositios y recursos tróficos de los que dependen las distintas especies de insectos saproxílicos. De manera que, una mayor proporción de oquedades voluminosas propicia una mayor diversidad de especies y complejidad de interacciones, incrementando así la estabilidad de los ensambles saproxílicos frente a la pérdida de microhábitats (Tabla 1) (Quinto et al., 2014, 2015).
Como era de esperar, distintos escenarios de eliminación de oquedades traen consigo distintas reorganizaciones de la diversidad de especies e interacciones, así como la variación de los principales atributos de interacción (Tabla 1). La extinción de las oquedades menos conectadas y la extinción aleatoria de oquedades pueden considerarse como escenarios de amenaza moderada, ya que la estabilidad de los ensambles saproxílicos no se ve excesivamente afectada ante la pérdida simulada del $50 \%$ de las oquedades. Contrariamente, los ensambles saproxílicos presentan una marcada sensibilidad ante la eliminación simulada de las oquedades más conectadas (las más voluminosas). Cabe subrayar que este tipo de simulación representa un escenario crítico pero factible, como puede ser la poda indiscriminada de los árboles viejos, lo que supondría un duro golpe a la estabilidad de los ensambles saproxílicos de los bosques mediterráneos (Quinto et al., 2015).

Estos resultados nos indican que los bosques mediterráneos estudiados en Cabañeros gozan actualmente de una buena disponibilidad de oquedades, lo que está vinculado al buen estado de conservación de los ensambles de insectos saproxílicos y su capacidad para amortiguar las perturbaciones.

Por otra parte, la composición y las características de las oquedades deben ser consideradas como herramientas valiosas para identificar ensambles potencialmente ricos, así como para el establecimiento de áreas de conservación prioritaria, prestando especial énfasis en la composición de oquedades voluminosas. Además, las oquedades arbóreas que actualmente son pequeñas y menos ricas en especies saproxílicas son asimismo importantes, pues aseguran el recambio a largo plazo de oquedades de los bosques mediterráneos, por lo que también deben ser tenidas en cuenta en programas específicos de conservación. Siendo así, las estrategias de conservación de las comunidades saproxílicas en los boques mediterráneos de la Península Ibérica deben contemplar criterios ambientales fundamentales para este grupo funcional, como son la estructura del bosque y la composición de microhábitats. 


\begin{tabular}{lc|c|c|c|c|c|c|c|c|c|c|c} 
& Robledal & R1 & R2 & R3 & Fresneda & F1 & F2 & F3 & Encinar & E1 & E2 & E3 \\
\hline Riqueza & 137 & 75 & 125 & 105 & 114 & 49 & 102 & 90 & 85 & 40 & 77 & 57 \\
\hline Abundancia & 2343 & 631 & 1712 & 1437 & 1219 & 345 & 909 & 590 & 825 & 211 & 614 & 471 \\
\hline $\begin{array}{l}\text { Enlaces por } \\
\text { especie }\end{array}$ & 3.76 & 2.26 & 3.03 & 2.63 & 2.75 & 1.76 & 2.47 & 2.01 & 2.4 & 1.38 & 2.16 & 2.01 \\
\hline Conectividad & 0.15 & 0.18 & 0.23 & 0.2 & 0.13 & 0.16 & 0.2 & 0.17 & 0.11 & 0.13 & 0.17 & 0.17 \\
\hline $\begin{array}{l}\text { Densidad de } \\
\text { enlaces }\end{array}$ & 10.58 & 5.63 & 10.62 & 8.3 & 8.64 & 3.9 & 9.07 & 8.39 & 7.3 & 3.38 & 7.18 & 6.83
\end{tabular}

Tabla 1. Principales atributos de interacción oquedad-saproxílico para cada bosque. R: robledal; F: fresneda; E: encinar; 1: eliminación del 50\% de las oquedades más conectadas; 2: eliminación del 50\% de las oquedades menos conectadas; 3: eliminación aleatoria del 50\% de las oquedades.

\section{REFERENCIAS}

Alexander, K.N.A. 2008. Tree biology and saproxylic Coleoptera: Issues of definitions and conservation language. Rev. Ecol. (Terre Vie). 63:1-5.

Dormann C.F., Gruber, B., \& Fründ, J. 2008. Introducing the bipartite Package: Analysing Ecological Networks. $R$ news 8:8-11.

García-lópez, A., Micó, E., Allemand, R., Alonso-Zarazaga M.A., Blas, M., Brustel, H., Galante, E., Herrmann, A., Leblanc, P., Lencina, J.L., Liberti, G., Marcos-García, M.A., Noblecourt, T., Otero, C., Quinto, J., Riba, J.M., Ricarte, A., Rose, O., Rotheray, G., Soldati, F., Tamisier, J.P., Verdugo, A., Vienna, P.P., Viñolas, A. \& Zapata de la Vega, J.L. 2014. Diversidad de coleópteros y dípteros (Syrphidae) saproxílicos del Parque Nacional de Cabañeros. En: Micó, E., Marcos-García, M.A. \& Galante, E. (eds.), Los insectos saproxílicos del Parque Nacional de Cabañeros. Organismo Autónomo de Parques Nacionales, Ministerio de Agricultura, Alimentación y Medio Ambiente, Madrid. pp. 71-111.

Haslett, J.R. 2007. European Strategy for the conservation of invertebrates. Nature and environment, No. 145.

Marcos-García, M.A., Micó, E., Quinto, J., Briones, R. \& Galante, E. 2010. Lo que las oquedades esconden. Cuadernos de Biodiversidad 34:3-7.
Marcos-García, M.A. \& Quinto, J. 2011. Mallota dusmeti Andréu, 1926. En: Verdú, J.R. \& Galante, E. (eds.), Atlas y Libro Rojo de los Invertebrados Amenazados de España. Especies Vulnerables (Vol. I). Ministerio de Medio Ambiente, Medio rural y Marino, Madrid. pp. 360-364.

Micó, E., García, A., Brustel, H., Padilla, A. \& Galante, E. 2013. Explaining the saproxylic beetle diversity of a protected Mediterranean area. Biodivers. Conserv. 22:889-904.

Micó, E., Marcos-García, M.A. \& Galante E. 2013. Los insectos saproxílicos del Parque Nacional de Cabañeros. Organismo Autónomo de Parques Nacionales, Ministerio de Agricultura, Alimentación y Medio Ambiente. Madrid. 139 pp.

Nieto, A. \& Alexander, K.N.A. 2010. European Red List of Saproxylic Beetles. Luxembourg: Publications Office of the European Union.

Quinto, J., Marcos-García, M.A. Brustel, H., Galante, E. \& Micó, E. 2013. Effectiveness of three sampling methods to survey saproxylic beetle assemblages. J. Insect Conserv. 17:765-776.

Quinto, J., Marcos-García, M.A. Díaz-Castelazo, C. RicoGray, V. Brustel, H., Galante E. \& Micó, E. 2012. Breaking down complex saproxylic communities: Understanding subnetworks structure and implications to network robustness. PLoS ONE 7:e45062. 
Quinto, J., Marcos-García, M.A., Díaz-Castelazo, C., RicoGray, V., Galante E. \& MICÓ, E. 2015. Association patterns in saproxylic insect networks among three Iberian Mediterranean woodlands and their resistance to microhabitat loss. PLoS ONE 10:e122141.

Quinto, J., Micó E., Marcos-García, M.A., Martínez-Falcón, A.P. \& Galante, E. 2014. Influence of tree hollow microenvironmental variables on saproxylic guild diversity in Iberian Mediterranean woodland. J. Insect Conserv. 18:981-992.
Verdú, J.R. \& Galante. E. 2006. Libro Rojo de los Invertebrados de España. Dirección General de Conservación de la Naturaleza. Ministerio de Medio Ambiente, Madrid.

Speight, M.C.D. 1989. Saproxylic invertebrates and their conservation. Nature and environment, series 42 . Council of Europe, Strasbourg. 